\title{
Caractérisation expérimentale du comportement hydroacoustique de turbomachines hydrauliques
}

\author{
Experimental characterization of the hydroacoustic behavior \\ of hydraulic turbomachines
}

par G. Caignaert, J. Charley

Laboratoire de Mécanique de Lille (URA CNRS 1441), ENSAM

This paper deals with the methods used in order to characterize a machine through a hydroacoustic transfer matrix and source terms. Intensimetry techniques in rectilinear ducts, which give these elements, are presented and discussed. These techniques use various plane waves propagation models which take into account the interactions between fluid flow and duct wall.

\section{INTRODUCTION}

L'endommagement des circuits hydrauliques et de certains composants de ces circuits, telles les pompes ou les vannes, a souvent pour origine des phénomènes vibratoires directement associés aux excitations issues des fluctuations de pression qui se propagent dans ces circuits constitués d'éléments passifs (éléments de transmission des perturbations) et actifs (éléments de transmission et de source de perturbations). Cette nature propagative des phénomènes conduit souvent à constater des dégradations de composants de circuits fort éloignés des sources principales de perturbations. Il est clair qu'on a toujours à considérer les mécanismes d'interactions entre le fluide véhiculé dans ces circuits et les structures qui le contiennent pour analyser les phénomènes observés. Il en est de même pour toutes les études visant à analyser ou à réduire le bruit de ces installations en général fort complexes.

La présente communication est limitée aux outils d'analyse expérimentale de ces phénomènes propagatifs dans les circuits hydrauliques dans le domaine des basses fréquences pour lesquelles il demeure légitime, compte tenu des longueurs d'ondes importantes vis-à-vis des dimensions transversales des circuits, de se limiter à l'hypothèse de propagation par ondes planes et de limiter les études vibratoires dans les circuits à des modèles de poutres.

De nombreux travaux ont été réalisés durant les quinze dernières années sur ces problèmes et ont vu se mettre en place les modèles de propagation et les techniques d'analyse expérimentale. La Société Hydrotechnique de France, à travers l'un des groupes de travail de sa division « applications de la mécanique des fluides ", a joué un rôle important à ce niveau et a permis de nombreux travaux en commun entre laboratoires industriels et universitaires, en particulier dans le domaine des pompes centrifuges, avec la constitution de bases de données hydroacoustiques relatives à trois machines aux géométries accessibles à tous. Beaucoup des résultats de ces travaux ont fait, entre autres, l'objet de présentations lors de colloques organisés par la SHF :

- sessions du comité technique 127 et 134 du 21 mars 1985 et des 17 et 18 juin 1988 [1, 2],

- $20^{\mathrm{c}}$ journées de l'hydraulique à Lyon en avril 1989 ,

- colloque «bruit et vibrations des pompes » de juillet 1993 [3],

- conférence sur le « comportement dynamique des pompes et turbines hydrauliques " en novembre 97 [4].

Ces travaux se sont tout d'abord attachés à la mise au point, d'une part de méthodes de caractérisation des régimes d'ondes de pression et de débit se propageant dans les circuits (méthodes intensimétriques multi-capteurs) et d'autre part de dispositifs visant à limiter les taux d'ondes stationnaires dans ces mêmes conduites de mesures (terminaisons anéchoïques pour liquides développées au CETIM). Les principes de représentation d'une turbomachine hydraulique ou de tout autre composant de circuit par une matrice de transfert hydroacoustique et des termes sources ont été mis en place depuis de nombreuses années et les approches expérimentales ont vu leur intérêt renforcé ces dernières années du fait du développement de codes de calculs de comportement hydro- et vibro-acoustique de circuits hydrauliques complexes fondés sur des principes équivalents (codes CIRCUS d'EDF [5] et LACTUS [6] par exemple). L'utilisation de ces codes requiert l'introduction des informations qui 
permettent de représenter le comportement des divers composants : pour des éléments complexes, telles les pompes ou les turbines en fonctionnement, cette caractérisation n'est à ce jour accessible qu'expérimentalement. Il faut noter que des techniques équivalentes sont utilisées pour la caractérisation aéro-acoustique d'éléments de circuits aérauliques (ventilateurs, tubulures d'échappement ou d'admission de moteurs, ...[7, 8]).

\section{MATRICE DE TRANSFERT HYDROA- COUSTIQUE}

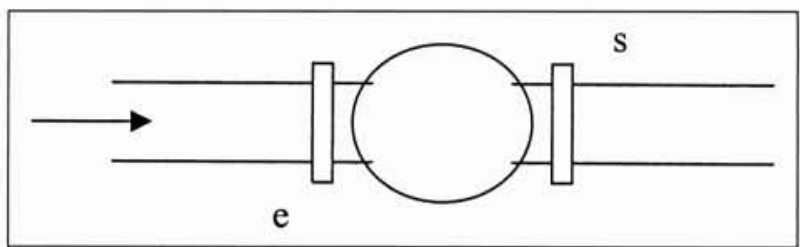

1. Représentation schématique d'une machine dans un circuit.

La figure 1 représente schématiquement une turbomachine hydraulique et ses conduites d'aspiration et de refoulement. Les caractéristiques hydroacoustiques de la machine relient les fluctuations de pression et de débit aux brides d'entrée (repère $e$ ) et de sortie (repère $s$ ). Si l'on ne prend pas en compte, dans un premier temps, les aspects sources, diverses formulations peuvent être utilisées pour représenter la transmission des perturbations à travers la machine.

En notant $p$ et $q$ les transformées de Fourier des fluctuations de pression et de débit masse, on peut par exemple écrire :

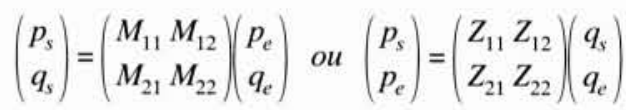

Les matrices $(M)$ et $(Z)$ sont dites respectivement matrice de transfert hydroacoustique et matrice d'impédance [9]. Les quatre termes de l'une ou l'autre de ces matrices sont des fonctions complexes de la fréquence $f$ et constituent les inconnues lors de la détermination expérimentale de ces caractéristiques de transfert. Les résultats obtenus peuvent être analysés soit pour caractériser le système étudié, soit pour en apprécier la qualité : c'est ainsi que le système peut être considéré comme symétrique, réciproque ou encore quasi-réciproque $[9,10]$.

- Un système symétrique est ainsi caractérisé par :

$$
\operatorname{det}(M)=1 \text { et } M_{11}=M_{22}
$$

- Un système réciproque est caractérisé par :

$$
\operatorname{det}(M)=1
$$

- Enfin, un système quasi-réciproque est défini par :

$$
|\operatorname{det}(M)|=1
$$

Dans le cas où le système étudié est non plus passif mais actif, c'est-à-dire générateur de perturbations, des termes sources sont pris en compte dans la formulation matricielle globale. L'une des formulations les plus couramment utilisées s'écrit, en considérant la source localisée ponctuellement dans le circuit :

$$
\left(\begin{array}{c}
p_{s} \\
q_{s}
\end{array}\right)=\left(\begin{array}{ll}
M_{11} & M_{12} \\
M_{21} & M_{22}
\end{array}\right)\left(\begin{array}{c}
p_{e} \\
q_{e}
\end{array}\right)+\left(\begin{array}{c}
\Delta p \\
\Delta q
\end{array}\right)
$$

La détermination expérimentale des termes de la matrice de transfert et des termes sources nécessite donc de disposer d'au minimum six informations indépendantes qui peuvent être obtenues par la réalisation d'au minimum trois essais du système étudié avec des conditions aux limites différentes du point de vue de l'hydroacoustique. Le plus souvent, on réalise deux essais en utilisant des sources de bruit extérieures au système étudié, de niveaux suffisants pour que le bruit propre généré par le système étudié puisse être négligé : on détermine alors la matrice de transfert. Puis on réalise un essai sans source extérieure, ce qui permet d'obtenir les deux termes sources. Divers types d'excitatrices extérieures ont été développés en fonction des gammes de fréquences visées. L'une des difficultés couramment rencontrées pour ce type d'essais provient des éventuels couplages vibratoires qu'il est difficile d'éliminer complètement compte tenu des importants niveaux d'excitation à atteindre. Il est par ailleurs très difficile d'obtenir des niveaux d'excitation suffisants sur une large gamme de fréquences compte tenu des caractéristiques propres de l'installation d'essais.

\section{III — INTENSIMÉTRIE EN CONDUITE}

Pour chacun des trois essais cités ci-dessus, il est donc nécessaire de savoir définir, pour chaque fréquence du domaine d'étude, les vecteurs d'état hydroacoustique en entrée et sortie de l'élément étudié. La mesure directe des fluctuations propagatives de débit demeurant très difficile à envisager, les techniques expérimentales reposent toutes pour l'instant sur des approches multi-capteurs dans les conduites rectilignes disposées à l'entrée et à la sortie de l'élément étudié. A l'heure actuelle, les laboratoires réalisant ces essais utilisent presque exclusivement des capteurs de fluctuations de pression montés en membrane affleurante dans des piquages réalisés sur les conduites. Les techniques de traitement les plus simples utilisent deux capteurs de pression pour la détermination d'un vecteur d'état dans la section droite se trouvant au milieu des deux capteurs et trois capteurs lorsqu'un contrôle de la célérité de propagation des ondes doit être effectué. Un transfert des informations obtenues vers la bride d'entrée ou de sortie du système étudié est ensuite effectué à l'aide d'un modèle de représentation du transfert hydroacoustique d'un élément de conduite rectiligne de section constante.

Dans le cas le plus élémentaire, c'est-à-dire en négligeant les effets des frottements du fluide, les effets du nombre de Mach de l'écoulement moyen et les vibrations de la paroi de la conduite, la matrice de transfert d'un élément de conduite de longueur $L$ s'écrit :

$$
\left(\begin{array}{l}
p_{2} \\
q_{2}
\end{array}\right)=\left[\begin{array}{cc}
\cos (k L) & -j \frac{\rho c}{S} \sin (k L) \\
-j \frac{S}{\rho c} \sin (k L) & \cos (k L)
\end{array}\right]\left(\begin{array}{l}
\left.p_{1}\right) \\
\left.q_{1}\right)
\end{array}\right.
$$

avec $k$ le nombre d'onde, $\rho$ la masse volumique du fluide, $c$ la célérité des ondes et $S$ l'aire de la section de passage.

Il est donc aisé, en s'appuyant sur une telle formulation, d'accéder à une caractérisation du vecteur d'état hydroacoustique dans la section milieu des deux sections extrémités où s'effectuent les mesures de fluctuations de pression. Expérimentalement, cela s'effectue par la détermination des autoet interspectres de fluctuations de pression avec évidemment la possibilité d'effectuer des moyennes sur plusieurs acquisitions de façon à améliorer la cohérence des informations recueillies. La détermination des vecteurs d'état en entrée et en sortie de la machine étudiée nécessite donc de bien utiliser un même capteur comme référence de phase pour l'ensemble des acquisitions. Par ailleurs, un étalonnage de bonne qualité des capteurs de pression utilisés et de leurs 
conditionneurs est donc important, tant en amplitude qu'en phase.

Pour contrôler la célérité de propagation des ondes étudiées, la technique maintenant couramment utilisée est celle qui a été proposée par Margolis et Brown [11] et qui utilise trois capteurs de pression en paroi de la conduite, l'un des capteurs se trouvant à égale distance $L$ des deux autres. En s'appuyant sur le modèle élémentaire défini par (6), il est aisé de démontrer que la demi-somme des fonctions de transfert $H$ des fluctuations de pression entre chaque capteur d'extrémité et le capteur central présente théoriquement l'allure de la fonction réelle suivante :

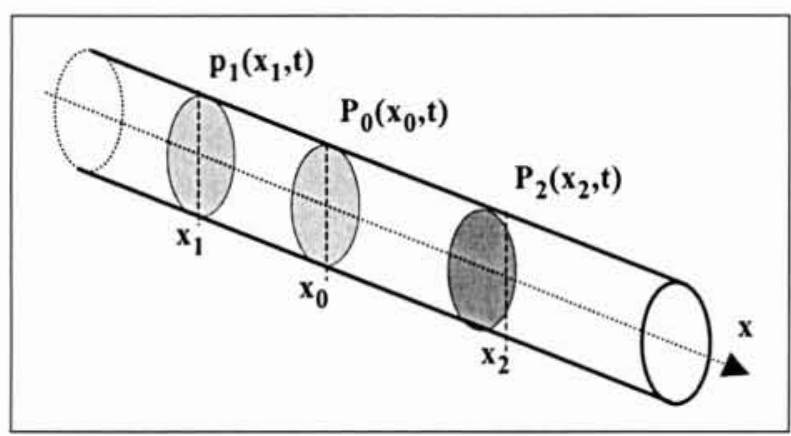

$$
\frac{1}{2}\left(H_{p_{1} p_{0}}+H_{p_{2} p_{0}}\right)=\cos k L
$$

La détermination expérimentale de cette demi somme et son identification à la relation (7) permettent de déterminer la célérité $c$ et de juger de la confiance à accorder au modèle utilisé. A ce niveau, les résultats obtenus permettent de rendre compte d'un couplage entre fluide et structure dans lequel le diamètre de la conduite varie sous l'effet des fluctuations de pression (modèle d'enveloppe mince avec divers types de conditions aux limites axiales). Les figures 2 et 3 illustrent deux résultats issus de la mise en œuvre de cette technique.

La distance entre capteurs doit être choisie avec soin en fonction de la gamme de fréquences à laquelle on s'inté- resse, sachant que de toute façon des singularités apparaissent nécessairement dans la procédure de calculs. La fréquence maximale d'utilisation de la méthode est par ailleurs limitée par l'apparition de modes de vibrations de la conduite ne permettant plus d'accepter l'hypothèse de propagation en ondes planes (modèles 1D, [12, 13]).

Lorsque la conduite étudiée présente un rapport épaisseur sur diamètre trop petit, il peut devenir nécessaire de prendre en compte les modes de vibrations de poutre pour caractériser plus exactement les propagations des perturbations $[14,15]$. Dans l'hypothèse d'un modèle de poutre de type Euler-Bernoulli, il apparaît un découplage dans le système d'équations à traiter. Les équations relatives aux moments de flexion, aux efforts tranchants, au moment de torsion, aux composantes transversales du déplacement du barycentre $G$ d'une section droite de poutre et aux rotations de cette section droite, sont découplées des quatre équations relatives à la pression dans le fluide, au débit de masse, à l'effort normal et à la composante axiale du déplacement de $G$. Seules ces quatre équations doivent donc être traitées et débouchent sur une formulation (en l'absence de frottements fluide et en négligeant l'effet du nombre de $\mathrm{MACH}$ de l'écoulement moyen en conduite) d'une matrice de transfert adimensionnée d'un élément de conduite de longueur $L$ de dimension 4 :

$$
\left\{X_{2}\right\}=\left(\begin{array}{c}
p_{2} \\
\rho_{f} c v_{2} \\
\frac{N_{2}}{S_{p}} \\
\rho_{p} g u_{x 2}
\end{array}\right)=[A] \cdot[B] \cdot[A]^{-1} \cdot\left\{X_{1}\right\}=\left[T_{4}\right]\left\{X_{1}\right\}
$$

avec :

$$
[B]=\left[\begin{array}{cccc}
e^{j r_{1} L} & 0 & 0 & 0 \\
0 & e^{j r_{2} L} & 0 & 0 \\
0 & 0 & e^{j r_{3} L} & 0 \\
0 & 0 & 0 & e^{j r_{4} L}
\end{array}\right]
$$

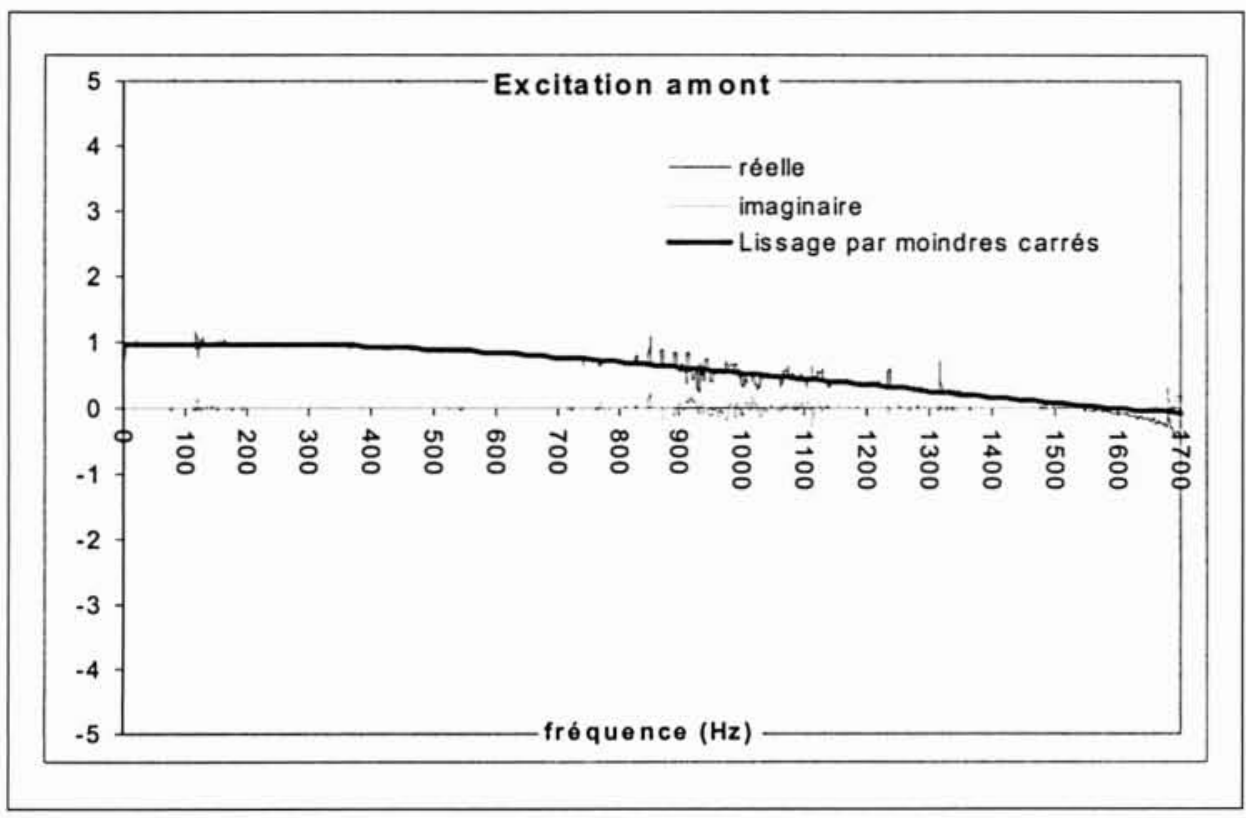

2. Détermination de la célérité dans une conduite en acier inoxydable de diamètre intérieur $44 \mathrm{~mm}$ et d'épaisseur $2,1 \mathrm{~mm}$. Méthode à 3 capteurs avec une distance entre capteurs égale à $200 \mathrm{~mm}$; essais en eau Célérité identifiée = $1230 \mathrm{~m} / \mathrm{s}$. 


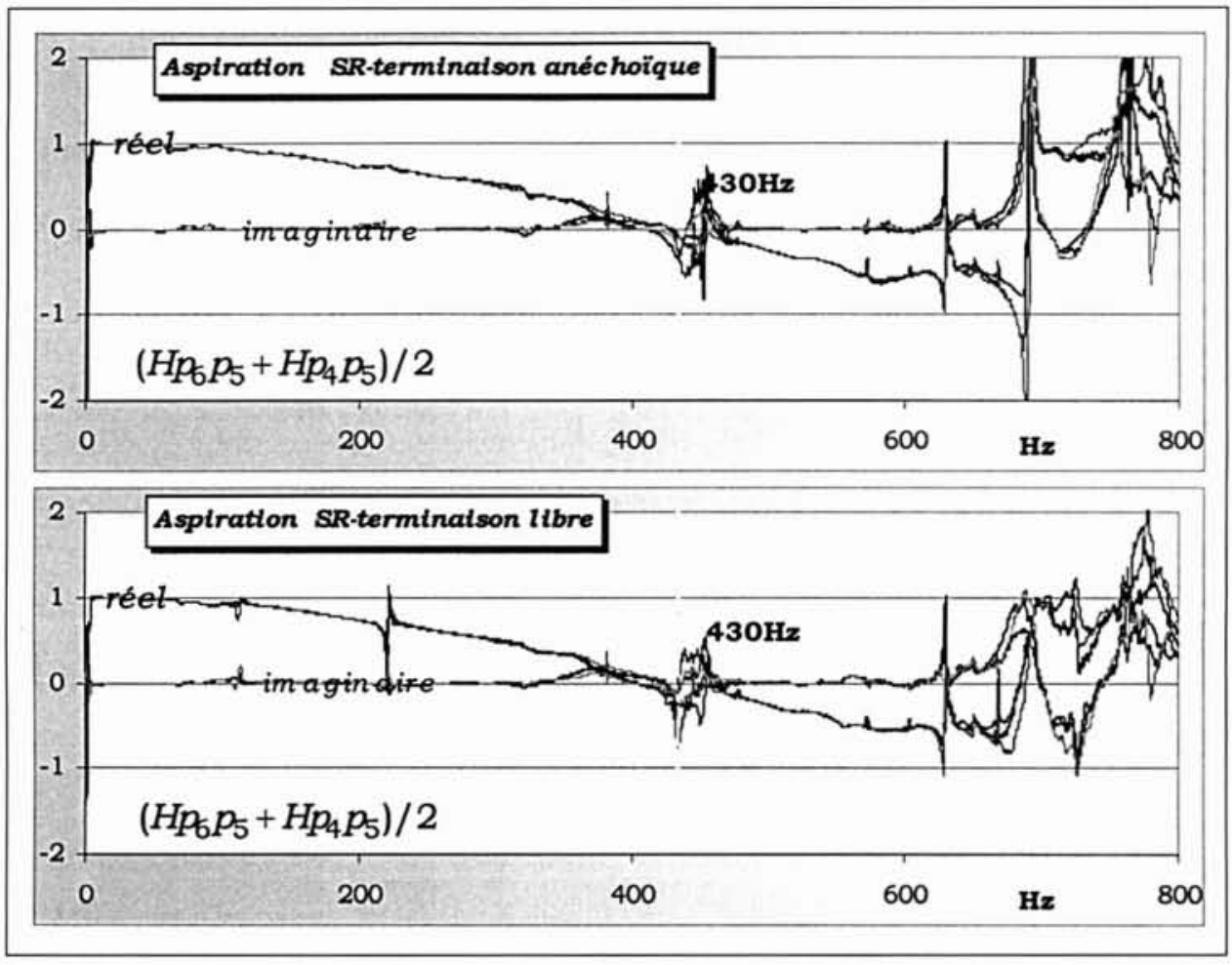

3. Détermination de la célérité dans une conduite en plexiglas de diamètre intérieur $292 \mathrm{~mm}$ et d'épaisseur 4 mm.

Méthode à 3 capteurs avec une distance entre capteurs égale à 200 mm ; essais en air. Célérité identifiée $=344$ $\mathbf{m} / \mathbf{s}$ (d'après la référence [17]).

et $[A]$ dont chaque colonne est définie par :

$$
\left(1 \frac{-r_{i}}{\rho_{f} \omega} \frac{S_{p} \frac{D v}{2 e}}{\frac{E r_{i}^{2}}{\rho_{p} \omega^{2}}-1} \frac{j r_{i} \frac{D v}{2 e}}{E r_{i}^{2}-\rho_{p} \omega^{2}}\right)^{t}
$$

dont les racines $r_{i}$ sont solutions de

$$
\left|\begin{array}{cccc}
r & -\rho_{p} S_{p} \omega^{2} & 0 & 0 \\
1 & S_{p} E r & S_{p} \frac{D}{2 e} v & 0 \\
0 & 0 & r & \rho_{f}\left(j \omega+v_{0} r\right) \\
0 & -2 v r\left(j \omega+v_{0} r\right) & \frac{1}{K}\left(j \omega+v_{0} r\right) & r
\end{array}\right|=0
$$

avec $D, e$ : diamètre et épaisseur de la conduite,

$E, v_{p}, S_{p}$ les propriétés du matériau et la section de la paroi de la conduite,

$\rho_{\rho} v_{0}$ la masse volumique du fluide et sa vitesse moyenne.

Du point de vue de l'expérimentateur, la prise en compte de ce couplage conduit à l'utilisation de quatre informations expérimentales pour accéder à la détermination d'un vecteur d'état dans une section quelconque de la conduite étudiée.

La figure 4, extraite de la référence [14], illustre des résultats obtenus dans une conduite en acier inoxydable de diamètre intérieur $100 \mathrm{~mm}$, d'épaisseur $2 \mathrm{~mm}$, parcourue par un écoulement d'eau à $2 \mathrm{~m} / \mathrm{s}$ dans un circuit d'essais de pompes, et munie de cinq capteurs de pression montés en membrane affleurante et régulièrement répartis le long de la tuyauterie (distance entre deux capteurs consécutifs égale à $500 \mathrm{~mm}$ ). On y voit une comparaison relative à la reconstitution de l'autospectre des fluctuations de pression au niveau du capteur central à l'aide d'un modèle inverse utilisant les informations expérimentales issues soit de deux des quatre autres capteurs (intensimétrie à deux capteurs) soit des quatre autres capteurs : il apparaît clairement sur cette figure que l'approche à quatre capteurs fournit un résultat de bien meilleure qualité si l'on fait référence à la valeur expérimentale.

Il est certain que ces méthodes intensimétriques utilisent presque exclusivement des capteurs de fluctuations de pression montés en membrane affleurante. Cela ne pose guère de difficulté sur une installation de laboratoire mais devient plus problématique lorsqu'il s'agit d'en envisager l'utilisation en environnement industriel. Des travaux sur la mise au point de méthodes non intrusives continuent à être développés et les travaux récemment entrepris par exemple au CETIM sur l'utilisation de fils de PDVF enroulés autour des tuyauteries semblent prometteurs.

\section{CONCLUSION}

La présente communication présente de façon assez rapide les approches expérimentales qui sont utilisées ou en cours de développement en vue de caractériser expérimentalement la matrice de transfert hydroacoustique et les termes sources associés au fonctionnement d'une turbomachine hydraulique (pompe ou turbine). Ces approches reposent sur l'identification des fluctuations de pression et de débit qui se propagent dans les conduites d'aspiration et de refoulement de ces machines. Cette identification fait appel à l'exploitation d'un nombre plus ou moins important d'informations expérimentales en fonction du modèle utilisé pour la mise en œuvre dans une méthode inverse : le couplage entre le fluide s'écoulant dans les tuyauteries et la paroi de ces tuyauteries peut ainsi être pris en compte à différents niveaux en fonction, essentiellement, de la valeur du rapport épaisseur sur diamètre et des caractéristiques élastiques respectives du fluide et du matériau de la conduite. Les modèles 1D ainsi 

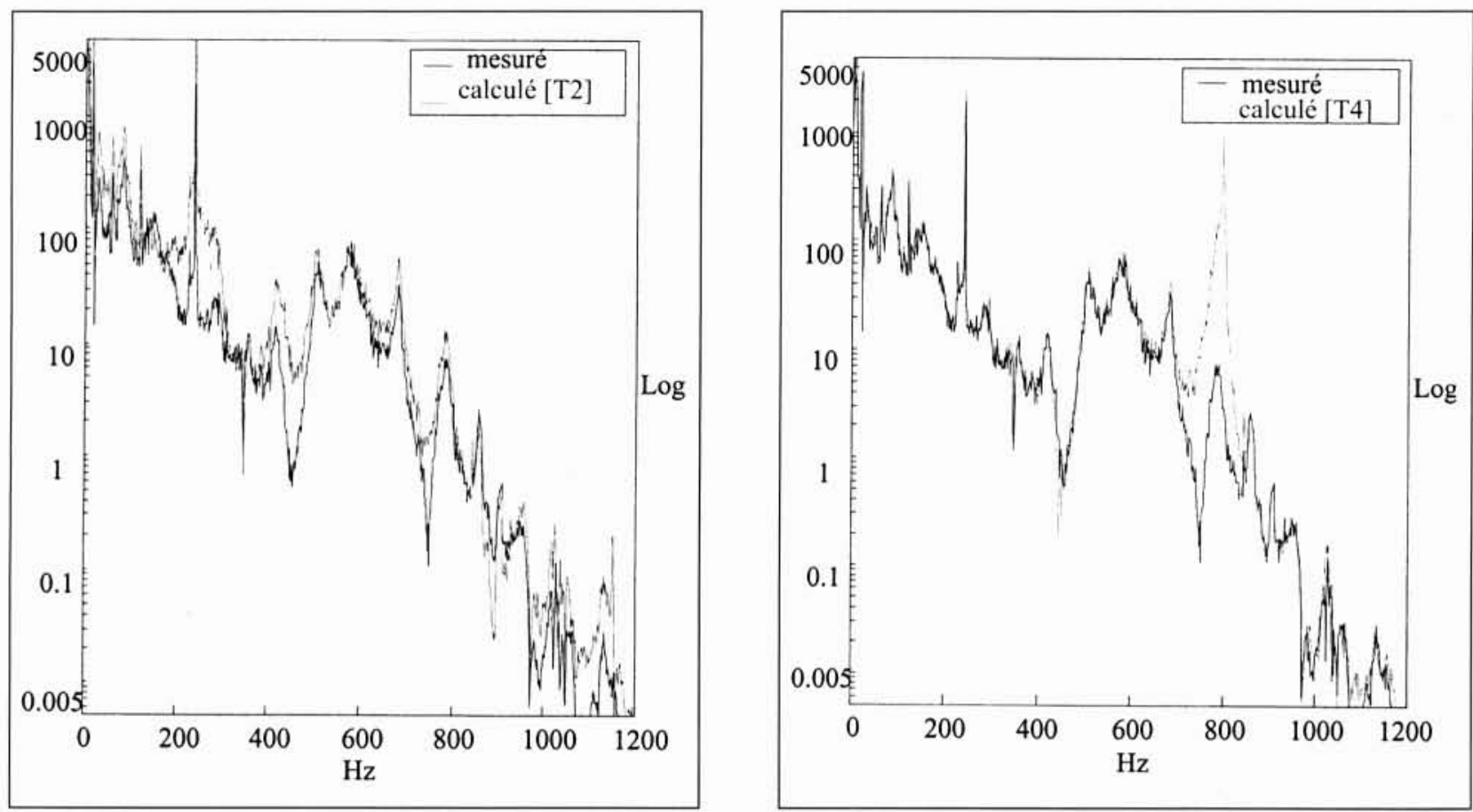

4. Comparaison des reconstitutions des autospectres des fluctuations de pression en un point d'une conduite obtenues avec des méthodes intensimétriques à deux et quatre capteurs avec le résultat expérimental directement mesuré. Comparaison en module (Log), Mesure (-), méthode 2 (----) et 4 capteurs (---).

mis en œuvre demeurent limités aux basses fréquences et ne peuvent plus être utilisés dès que l'on s'approche des premiers modes de vibrations de coques.

Des approches très voisines sont utilisées pour la caractérisation du comportement dynamique de circuits aérauliques (admission ou échappement de moteurs, ventilateurs, ...).

Plusieurs résultats expérimentaux $[16,17]$ relatifs à la caractérisation de la matrice de transfert de pompes centrifuges à simple volute, obtenus tant en air qu'en eau, indiquent que ces matrices apparaissent indépendantes des conditions de fonctionnement (hors cavitation) sur une large plage de fréquences, ce qui autorise l'utilisation de techniques plus rapides à mettre en œuvre pour effectuer cette caractérisation (utilisation de techniques impulsionnelles sur la machine à l'arrêt par exemple).

On semble maintenant aboutir à la possibilité de la mise en œuvre de normes de caractérisation comme il en existe par exemple pour certaines machines volumétriques [18], même si une réflexion importante mérite d'être poursuivie sur le choix des capteurs, leur étalonnage dynamique et les exigences en matière de précision qu'il est raisonnable d'atteindre. De même, une réflexion sur les exigences contractuelles admissibles en matière de fluctuations de pression générées par les turbomachines hydrauliques dans les circuits et sur la façon de formuler ces exigences doit être mise en chantier.

\section{RÉFÉRENCES}

[1] La Houille Blanche, $\mathrm{n}^{\circ} 8,1985$.

[2] La Houille Blanche, $\mathrm{n}^{\circ} 3 / 4,1988$.

[3] Bruit et Vibrations des Pompes, Publications CETIM, 1993.

[4] La Houille Blanche, $n^{\circ}$ 3/4, 1998.
[5] De Montussaint D., Trolle J.L., Villouvier V. (1993). - CIRCUS, code de calcul des vibrations de tuyauteries induites par les écoulements, Bruit et Vibrations des Pompes. Publications CETIM, pp. 329-336.

[6] Landel. E., Blanc P., DiCostanzo F. (1993). - LaCTUS : logiciel d'aide à la conception de réseaux de tuyauteries silencieux, Bruit et Vibrations des Pompes. Publications CETIM, pp. 313-320.

[7] Lavrentjev J., Авом M., Boden H. (1995). - A measurement method for determining the source data of acoustic two-port sources. Journal of Sound and Vibration, 183(3), pp. 517-531.

[8] Lavrentjev J., Авом H. (1995). - Characterization of fluid machines as acoustic multi-port sources. Journal of Sound and Vibration, 197(1), pp. 1-16.

[9] BREnNen C. (1994). - Hydrodynamics of Pumps. Oxford Science Publications (ISBN : 0-933283-07-5).

[10] BRown F.T. (1967). - A unified approach of the analysis of uniform one-dimensional distributed systems. ASME Journal of Basic Engineering, June, pp. 423-432.

[11] MARgolis D.L., Brown F.T. (1976). - Measurements of the propagation of long-wavelength disturbances through turbulent flow in tubes. ASME Journal of Fluids Engineering, March, vol. 98 , pp. 70-75.

[12] Акоuм M. (1997). - Méthode de mesure des matrices de réflexion et transmission en pression acoustique, liées aux discontinuités dans les conduits cylindriques. Thèse U.T. Compiègne, mars.

[13] Tephany F., Thomas P. (1987). - Vibrations des tuyauteries sous écoulement : premières analyses. EDF, bulletin DER série A, 1987, n 4, pp. 33-49.

[14] Charley J. (1993). - Intensimétrie en conduite, prise en compte du couplage fluide-structure. Thèse de l' ENSAM, décembre. 


\section{lécanique des fluides et applications}

[15] DE JoNG. - Analysis of pulsations and vibrations in fluidfilled pipe systems. PHD Thesis, Eindhoven University of Technology. TNO Institute of Applied Physics, Delft.

[16] Bolpaire S., BarRand J.P. (1999). - Hydroacoustical study of a centrifugal pump. IAHR Work Group on the bahavoir of hydraulic machinery undre steady oscillatory conditions ; BRNO (Czech Republic) 7-9 September (paper B6; 12 pages)
[17] Charley J., Carta F., ABdallahi M.O. (1998), - Vibroacoustical analysis of flow in piping system, SME/JSME Joint Pressure Vessels and Piping Conference. Advances in fluids, structures, and fluid/structures interactions, July 2630, San Diego (PVP-Vol. 377-1, pp. 245-252).

[18] Norme ISO 10767 (1996). - Transmissions hydrauliques. Détermination des niveaux d'onde de pression engendrés dans les circuits et composants. 